\title{
COMPACT EXTENSIONS OF COMPACTLY GENERATED NILPOTENT GROUPS ARE PRO-LIE
}

\author{
KARL H. HOFMANN, JOHN R. LIUKKONEN AND MICHAEL W. MISLOVE
}

\begin{abstract}
We show that compact extensions of compactly generated (locally compact) pro-Lie groups are pro-Lie, and that compactly generated (locally compact) nilpotent groups are pro-Lie. As a consequence compact extensions of compactly generated nilpotents are pro-Lie. We give examples indicating limitations to extending our results.
\end{abstract}

0. Introduction. In work which culminated in [5], the Fourier-Stieltjes algebra $B(G)$ of certain locally compact groups $G$ was studied. The groups in question were all compact modulo a closed normal nilpotent subgroup, and at first results were obtained only for such groups which in addition were either Lie groups, or else were compactly generated. It then became clear that these results could be simplified if all such groups could be shown to be pro-Lie, and that is the purpose of the present paper.

To fix notation, we consider locally compact groups $G$ with a closed normal subgroup $N$ and quotient group $K=G / N$. We obtain various results leading up to the main point of the paper: First, if $N$ is pro-Lie and $K$ is compact, then the compact normal co-Lie subgroups of $N$ which intersect in (1) may be chosen to be $K$-invariant. If, on the other hand, $G$ is compactly generated, $K$ is pro-Lie, and $N$ is a compactly generated central subgroup of $G$, then $G$ is again pro-Lie. Finally, if $K$ is compact and $N$ is a compactly generated pro-Lie group, then $G$ is also pro-Lie. These results are then used to establish our main result: If $N$ is compactly generated nilpotent and $K$ is compact, then $G$ is pro-Lie.

In order to put our results in historical perspective, we point out that the fundamental result that almost connected groups are pro-Lie fits into our setting, with $N=G_{0}$ (see [6, Theorem 4.6]). Grosser and Moskowitz [3, Theorem 2.11], extending a result of S. P. Wang, showed that $[S I N]_{B}$ groups are $B$ pro-Lie for compact groups $B$ containing the inner automorphism group of $G$. In particular, all $[Z]$ groups are pro-Lie.

The authors gratefully acknowledge the support of the National Science Foundation during the course of the research contained herein.

1. Definition. Suppose $G$ is a locally compact group and $B$ a group of automorphisms of $G$. Then $G$ is $B$ pro-Lie if there is a family $\left\{K_{\alpha}\right\}$ of compact normal

Received by the editors March 5, 1981.

1980 Mathematics Subject Classification. Primary 22D05; Secondary 20F18.

Key words and phrases. Pro-Lie, nilpotent locally compact group, compactly generated locally compact group. 
$B$-invariant subgroups of $G$ such that $G / K_{\alpha}$ is Lie for each $\alpha$, and $\cap K_{\alpha}=(1)$. If $B=(1)$, then $G$ is pro-Lie.

The following two results are very similar to Theorem 2.11 of [3]; we include a proof for completeness.

2. Proposition. Suppose $G$ is a pro-Lie group and B a compact group of automorphisms of $G$. Then $G$ is $B$ pro-Lie.

(Note: We mean that $B$ is compact as a subgroup of $\operatorname{Aut}(G)$ or, equivalently, in the compact-open topology. For a summary of this topology, see [1].)

Proposition 2 is an immediate corollary of Lemma 3 below.

3. LeMma. Suppose $K$ is a compact normal subgroup of the locally compact group $G$ with $G / K$ Lie. If $B$ is a compact group of automorphisms of $G$ and $N=\cap\{\beta K$ : $\beta \in B\}$, then $G / N$ is Lie.

Proof. We show that $N$ is in fact the intersection of finitely many images $\beta K$, and the result then follows. First, since $G / K$ is Lie, $G / K$ has no small subgroups, and so there is a neighborhood $U$ of $K$ in $G$ so that any subgroup $L$ of $G$ with $L \subseteq U$ satisfies $L \subseteq K$. Thus $S=\{\beta \in B: \beta K \subseteq K\}$ is open in $B$. Now, $S \cap S^{-1}$ is also open in $B$, and in fact $S \cap S^{-1}=\{\beta \in B: \beta K=K\}$. Hence the $B$-orbit of $K$ is finite (as $B$ is compact), and so $N=\bigcap\{\beta K: \beta \in B\}$ is a finite intersection.

4. TheOREM. Let $G$ be a locally compact group, and let $N$ be a compactly generated closed normal pro-Lie subgroup such that $K=G / N$ is compact. Then $G$ is pro-Lie.

Proof. Let $K_{\alpha}$ be an arbitrary compact normal subgroup of $N$ such that $N_{\alpha}=N / K_{\alpha}$ is Lie. By Proposition 2 and Lemma 3, we may assume with no loss of generality that $K_{\alpha}$ is $G$-normal. For the moment replace $G$ by $G / K_{\alpha}$, and $N$ by $N_{\alpha}$; i.e., assume temporarily that $N$ is Lie.

We first observe that $\operatorname{Aut}(N)$ is a Lie group. In fact, by looking at the restriction map $r: \operatorname{Aut}(N) \rightarrow \operatorname{Aut}\left(N_{0}\right)$, we see that $\operatorname{Aut}(N) / \operatorname{ker} r$ is Lie, and $\operatorname{ker} r \subset \operatorname{Aut}\left(N / N_{0}\right)$ is discrete since $N / N_{0}$ is finitely generated. So $\operatorname{Aut}(N)$ is Lie. It then follows that $G / Z(N, G)$ is Lie, where $Z(N, G)$ is the centralizer of $N$ in $G$. This is because $Z(N, G)$ is the kernel of the map $G \rightarrow \operatorname{Aut}(N)$. But $G$ acts on $Z(N, G)$ through the compact group $G / N$, and so by [1, Theorem 4.1], $Z(N, G) \in[S I N]_{G}$, and by [3, Theorem 2.11], $Z(N, G)$ is $G$ pro-Lie.

This shows that $G / K_{\alpha}$ is pro-Lie, and since $K_{\alpha}$ was arbitrarily small, it follows $G$ is pro-Lie.

5. Lemma. Supposed (1) $\rightarrow D \rightarrow K \rightarrow Q \rightarrow$ (1) is a quotient exact sequence, where $K$ is compact, and D is a Lie subgroup of the center of $K$. Suppose $G$ is a group of automorphisms of $K$ which fixes $D$ pointwise, and suppose $K / D$ is $G$ pro-Lie. Then there are a closed subgroup $S$ of $K$ and a closed $G$-invariant normal subgroup $T$ of $K$ such that $S \cap D=(1), T=S \times D$ and $K / T$ is a Lie group.

Proof. Since $D$ has no small subgroups, we can find a neighborhood $U$ of 1 in $K$ such that if $M \subset U$ is a subgroup, then $M \cap D=(1)$. Since $K$ is pro-Lie, we can 
now find a $K$-normal closed subgroup $P$ of $K$ such that $K / P$ is Lie and $P \subset U$, so $P \cap D=(1)$. Now $K / P D$ is Lie, and so there exists a neighborhood $W$ of $P D$ such that if $M \subset K$ is a subgroup with $M \subset W$, then $M \subset P D$. Now since $Q=K / D$ is $G$ pro-Lie, there is a $G$-invariant closed normal subgroup $T$ of $K$ such that $D \subset T \subset W$ and $K / T$ is Lie. So we have $D \subset T \subset P D$. Let $S=P \cap T$.

We claim $T=S \times D$. In fact, if $t \in T$, then $t \in P D$, so $t=p d$ where $p \in P$ and $d \in D$. But then $p=t d^{-1} \in T D \subset T$, so $p \in S$. Thus $t \in S D$. This shows $T=S D$. But $S \cap D=(1)$, so $T=S \times D$.

6. Lemma. Suppose $S$ is a compact group and $D$ a compact abelian Lie group. Suppose $G$ is a compactly generated group of automorphisms of $T=S \times D$ which fixes $D$ pointwise and is such that $T / D$ is $G$ pro-Lie. Then there is a compact G-invariant normal subgroup $M$ of $T$ such that $T / M$ is Lie and $M \cap D=(1)$.

Proof. Without loss of generality, we may replace $T$ by $T /[T, T]^{-}$and assume thereby that $S$ is abelian. Our given hypotheses are: $S$ is compact abelian, $D$ compact Lie abelian, $G$ a compactly generated group of automorphisms of $T=S \times$ $D$ which fixes $D$ pointwise, such that $T / D$ is $G$ pro-Lie. Our goal is to find a closed normal $G$-invariant subgroup $M$ of $T$ such that $T / M$ is Lie and $M \cap D=(1)$.

We solve this problem by dualizing: we let $C=\hat{T}, A=(T / D) \hat{)} \simeq \hat{S}$, and $B=$ $(T / S) \simeq \hat{D}$. Then we have $C=A \times B ; A, B$, and $C$ are discrete; $B$ is finitelygenerated (as the dual of the Lie group $D$ ). Moreover, $G$ is a compactly generated group of automorphisms of $C$, and $G$ preserves $A$ cosets (since $C / A \simeq B \simeq \hat{D}$ and $G$ fixes $D$ pointwise). Finally $A$ is the union of finitely-generated $G$-invariant subgroups (since $A=(T / D)$ and $T / D$ is $G$ pro-Lie). On the basis of all this, we want to find a finitely-generated $G$-invariant subgroup $E(=(T / M)$, where $M$ is the sought-for subgroup of $T$ ) of $C$ such that $A E=C$.

We claim that the smallest $G$-invariant subgroup of $C$ containing $B$ is finitelygenerated. (This will prove Lemma 6.) Since $B$ is finitely-generated and abelian, it is enough to show that the subgroup generated by $G b$ is finitely-generated for each $b \in B$. To see this, let $Y$ be a compact symmetric generating set in $G$. Then $Y b$ is finite in $C$, hence contained in $b+A_{0}$ for some finitely-generated $G$-invariant subgroup $A_{0}$ of $A$. Since $Y$ generates $G$, we clearly have $G b \subset b+A_{0}$. But the subgroup $E$ of $C$ generated by $G b$ is clearly contained in $\mathbf{Z} b+A_{0}$, so $E$ is finitely-generated, and Lemma 6 is proved.

7. TheOREM. Suppose (1) $\rightarrow R \rightarrow G \rightarrow H \rightarrow$ (1) is a quotient exact sequence. Suppose $H$ is pro-Lie, $R \subset Z(G)$, and $R$ and $G$ are both compactly generated. Then $G$ is pro-Lie.

Proof. To show $G$ is pro-Lie, it is enough to show that $\cap\{K \triangleleft G \mid K$ is compact and $G / K$ is Lie $\}=(1)$. In particular, we need to show there is a compact normal $K$ in $G$ such that $G / K$ is Lie.

Let $N$ be any compact normal subgroup of $H$ such that $H / N$ is Lie. Let $M$ be the pre-image of $N$ in $G$. Thus $M \in[Z]$ is compactly generated and so $M$ contains a maximum compact subgroup $C$, and $M / C$ is a Lie group [1, esp. Corollary 1, p. 
336]. Now $C$ must be normal in $G$, and $G / C$ is Lie, since $G / M(\simeq H / N)$ and $M / C$ are both Lie. This shows that $\cap\{K \triangleleft G \mid K$ is compact and $G / K$ is Lie $\} \subseteq M$. Since $H$ is pro-Lie, and $N$ is an arbitrary compact normal subgroup of $H$ such that $H / N$ is Lie, we get $\cap\{K \triangleleft G \mid K$ is compact and $G / K$ is Lie $\} \subset R$.

Now $R$ is compactly generated abelian, and so contains a maximum compact subgroup $D$. We now have $\cap\{K \triangleleft G \mid K$ is compact and $G / K$ is Lie $\} \subset D$. Thus we only need to find, for any compact subgroup $N$ of $D$ such that $D / N$ is Lie, a compact normal subgroup $M$ of $G$ such that $G / M$ is Lie and $M \cap D \subset N$. (We use here that $D$ is central and pro-Lie.) In fact, let $N$ be an arbitrary compact subgroup of $D$ such that $D / N$ is Lie, and replace $D$ by $D / N$. Thus we assume $D$ is Lie, and must find a compact normal subgroup $M$ of $G$ such that $G / M$ is Lie and $M \cap D=(1)$.

Now let $L$ be any compact normal subgroup of $G$ such that $G / L$ is Lie, and let $K=L D$. Then $K$ is compact normal in $G$ and $G / K$ is Lie. By Lemma 5 , there is a compact $G$-normal subgroup $T$ of $K$ such that $G / T$ is Lie and $T=S \times D$ for some closed $K$-normal subgroup $S$ of $K$. But then by Lemma 6, we have a compact normal subgroup $M$ of $G$ such that $G / M$ is Lie and $M \cap D=(1)$. Translating this conclusion back to before we divided by $N$, we can say that for any compact subgroup $N$ of $D$ such that $D / N$ is Lie, there is a compact normal subgroup $M$ of $G$ such that $G / M$ is Lie and $M \cap D \subset N$. Since $D$ is $G$ pro-Lie, we conclude $\cap\{K \triangleleft G \mid K$ is compact and $G / K$ is Lie $\}=(1)$; i.e., $G$ is pro-Lie.

8. Proposition. If $N$ is a compactly generated nilpotent locally compact group, then $Z(N)$ is compactly generated.

Proof. We denote the upper central series by

$$
1=Z^{0} \subset Z^{1} \subset \cdots \subset Z^{n}=N,
$$

and the lower central series by

$$
N=C^{0} \supset C^{1} \supset \cdots \supset C^{n}=1 .
$$

Recall that $C^{k}=\left[N, C^{k-1}\right]$ for $k=1, \ldots, n$, and that the lengths of the two series do agree [4, p. 2]. We are considering here commutators in the algebraic sense and do not claim any $C^{k}$ is necessarily closed.

We claim first that each $C^{k}$ is compactly generated modulo $C^{k+1}$ in the sense that each $C^{k}$ contains a compact subset which generates it modulo $C^{k+1}$. We prove this by induction on $k$. Letting $[x, y]=x y x^{-1} y^{-1}$ and $x^{y}=y^{-1} x y$, we have the easily verified relations

(a) $[x y, z]=[y, z]^{x^{-1}}[x, z]$ and

(b) $[x, y z]=[x, y][x, z]^{y^{-1}}$.

Let $X$ be a symmetric compact generating set for $N$ (i.e., $N=\langle X\rangle$, the smallest group containing $X)$. Then a typical generator for $C^{1}$ may be written $\left[x_{1} \ldots x_{m}, y_{1} \ldots y_{n}\right]$, where each $x_{i}$ and $y_{j} \in X$. By (a) and (b) this generator is in turn a product of elements of the form $\left[x_{i}, y_{j}\right]$, modulo $C^{2}$. This proves the claim for $k=1$. Now suppose $C^{k}$ is compactly generated modulo $C^{k+1}$; that is, for some 
symmetric compact set $W_{k} \subset C^{k}$, the subgroup $\left\langle W_{k}\right\rangle$ has the property $\left\langle W_{k}\right\rangle C^{k+1}=$ $C^{k}$. Then a typical generator of $C^{k+1}$ may be written $\left[x_{1} \ldots x_{m} t, y_{1} \ldots y_{n}\right]$, where each $x_{i} \in W_{k}, t \in C^{k+1}$, and each $y_{j} \in X$. But by (a) and (b) this element is the product of elements of the form $\left[x_{i}, y_{j}\right]$ and $\left[t, y_{j}\right]$, modulo $C^{k+2}$. This shows $C^{k+1}$ is compactly generated modulo $C^{k+2}$, so that the claim is proved by induction. It follows easily that each $C^{k}$ contains a compact generating set, and (since $N$ is locally compact) that each $\overline{C^{k}}$ is compactly generated.

Now we claim that $Z(N)$ is compactly generated. We prove this by induction on the (nilpotence) class of $N$. For $N$ of class one ( $N$ abelian) the assertion is trivial. Suppose the assertion holds for all compactly generated nilpotent groups of class $\leqslant n$. Let $N$ be a compactly generated nilpotent group of class $n+1$. Then $C^{n} \subset Z^{1}$ and $\overline{C^{n}} \subset Z^{1}$. But $N / \overline{C^{n}}$ is of class $\leqslant n$, and so $Z\left(N / \overline{C^{n}}\right)$ is compactly generated by inductive hypothesis. Now $Z^{1} / \overline{C^{n}}$ is a closed subgroup of the compactly generated locally compact abelian group $Z\left(N / \overline{C^{n}}\right)$ and hence is itself compactly generated. Hence $Z^{1}=Z(N)$ is compactly generated. This proves $Z(N)$ is compactly generated for compactly generated nilpotent $N$ of all classes, by induction.

9. ThEOREM. Let (1) $\rightarrow N \rightarrow G \rightarrow K \rightarrow$ (1) be a quotient exact sequence, where $N$ is compactly generated nilpotent and $K$ is compact. Then $G$ is pro-Lie.

Proof. By repeated application of Theorem 7 and Proposition 8 , we see by induction that $N$ is pro-Lie. But then $G$ is pro-Lie by Theorem 4 .

10. EXAmples. We present here some examples to indicate limitations on extending our results.

A. Let $G=\mathbf{T}^{\infty} \rtimes \mathbf{Z}$, where $\mathbf{T}^{\infty}$ is the infinite product of $\mathbf{T}$ indexed from $-\infty$ to $\infty$, and $\mathbf{Z}$ acts by powers of the bilateral shift. Then $G$ is a compactly generated solvable group which is not pro-Lie. Thus "nilpotent" seems necessary in Theorem 9.

B. Let $G$ be the Heisenberg group over the $p$-adic field. Then $G$ is nilpotent but not compactly generated. Also, $G$ is 0 -dimensional but not [SIN], hence not pro-Lie. So "compactly generated" is necessary in Theorem 9.

C. In this example we construct a class 2 nilpotent group $G$ with center $Z(G)=Z_{2}$ such that $G$ is not pro-Lie. This shows that in Theorem 7 we need to assume $G$ is compactly generated. We let $H=\mathbf{Z}_{2} \oplus \Sigma \mathbf{Z}_{2}$, and we let the compact group $K=\Pi \mathbf{Z}_{2}$ act on $H$ by:

$$
\left(\alpha_{n}\right)\left(x,\left(x_{n}\right)\right)=\left(x+\sum \alpha_{n} x_{n},\left(x_{n}\right)\right)
$$

Here we mean $\alpha_{n} x_{n}=0$ unless both $\alpha_{n}$ and $x_{n}=1$, in which case $\alpha_{n} x_{n}=1$. Then let $G=H \rtimes K$; we get $Z(G)=\mathbf{Z}_{2}$, and $G / Z(G)=\Sigma \mathbf{Z}_{2} \oplus \Pi \mathbf{Z}_{2}$.

D. If, following [2, p. 385], we let $G=\left(\Sigma \mathbf{Z}_{3}\right) \rtimes \Pi \mathbf{Z}_{2}$; where each $\mathbf{Z}_{2}$ acts on $\mathbf{Z}_{3}$ by inversion, then we get that $G$ is a compact extension of an infinitely-generated discrete group which is not pro-Lie. Thus it seems necessary in Theorem 4 that $N$ be compactly generated. 


\section{REFERENCES}

1. S. Grosser and M. Moskowitz, On central topological groups, Trans. Amer. Math. Soc. 127 (1967), 317-340.

2. __ Representation theory of central topological groups, Trans. Amer. Math. Soc. 129 (1967), $361-390$.

3. ___ Compactness conditions in topological groups, J. Reine Angew. Math. 246 (1971), 1-40.

4. P. Hall, The Edmonton notes on nilpotent groups, Queen Mary College Mathematics Notes.

5. J. R. Liukkonen and M. Mislove, Fourier-Stieltjes algebras of compact extensions of nilpotent groups, J. Reine Angew Math. 325 (1981), 210-220.

6. D. Montgomery and L. Zippin, Topological transformation groups, Interscience, New York, 1955.

Department of Mathematics, Tulane University, New Orleans, Louisiana 70118 